SANTOS, C.M.S. e SANTANA, A.F. Produção de leite e duração da lactação de cabras da raça Pardo-alpina no município de Amélia Rodrigues - BA. PUBVET, Londrina, V. 5, N. 25, Ed. 172, Art. 1162, 2011.

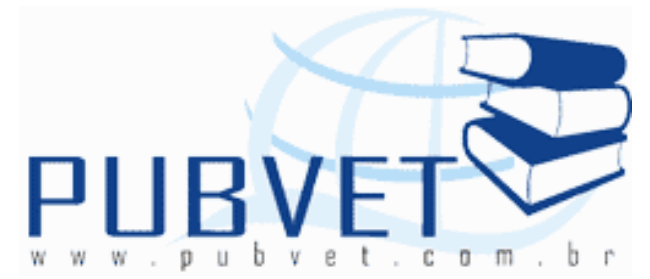

PUBVET, Publicações em Medicina Veterinária e Zootecnia.

\title{
Produção de leite e duração da lactação de cabras da raça Pardo-alpina no município de Amélia Rodrigues - BA
}

Charles Muller Silva dos Santos ${ }^{1}$ e Adelmo Ferreira de Santana ${ }^{2 *}$

${ }^{1}$ Estudante de Graduação da Escola de Medicina Veterinária da Universidade Federal da Bahia (UFBA)

${ }^{2}$ Médico Veterinário e Professor do Departamento de Produção Animal, UFBA.

Departamento de Produção Animal, Escola de Medicina Veterinária, Universidade Federal da Bahia (UFBA). Av.Ademar de Barros 500 Ondina 40170-110 - Salvador, BA - Brasil. E-mail: afs@ufba.com.br. *Autor para contato.

\section{Resumo}

Foram utilizadas 20 cabras da raça Pardo-alpina de um criatório localizado em Amélia Rodrigues - BA com o objetivo de avaliar a duração da lactação e produção diária e total de leite durante a primeira e segunda parição. $\mathrm{Na}$ primeira lactação a média de produção e duração foram 408,81 $\pm 35,697 \mathrm{Kg}$ de leite e 191,665 $\pm 20,907$ dias, respectivamente, enquanto que na segunda lactação a média de produção passou a ser 482,93 $\pm 65,917 \mathrm{Kg}$ de leite e duração de 219,630 \pm 28,047 dias, havendo alta correlação positiva entre essas duas variáveis. Porém não houve diferença significativa $(p>0,05)$ na produção diária entre as parições.

Palavras-chave: Leite de cabra, produção de leite, duração da lactação, cabras Pardo-alpina. 
SANTOS, C.M.S. e SANTANA, A.F. Produção de leite e duração da lactação de cabras da raça Pardo-alpina no município de Amélia Rodrigues - BA. PUBVET, Londrina, V. 5, N. 25, Ed. 172, Art. 1162, 2011.

\title{
Milk production and lactation length of Alpine goats on municipal district of Amélia Rodrigues - BA
}

\begin{abstract}
20 Pardo-alpina goats from a farm localizated in the municipal district of Amélia Rodrigues - BA were used to evaluate the lactation length and daily or total milk production during the first and second calving. In the first lactation the average of milk production and length was $408,81 \pm 35,697 \mathrm{Kg}$ of milk and $191,665 \pm 20,907$ days, respectively, while in the second lactation the average of production became $482,93 \pm 65,917 \mathrm{Kg}$ of milk and the length, 219,630 \pm 28,047 days. There was a high positive correlation behind milk production and lactation length. However there wasn't significative difference $(p>0,05)$ with the daily production behind the calvings.
\end{abstract}

Keywords: Milk of goat, milk production, lactation length, Alpine goats.

\section{INTRODUÇÃO}

A caprinocultura leiteira no Brasil, nas últimas três décadas, vem se consolidando como atividade rentável, despertando o interesse de muitos produtores rurais (GONÇALVES et al., 2002).

Segundo Quadros (2008) produção de leite de cabra pode se tornar um importante instrumento na política de alimentos diminuindo os níveis de subnutrição e taxa de mortalidade infantil em várias regiões, principalmente no nordeste brasileiro onde, apesar das condições climáticas adversas, os caprinos são mais adaptados e apresentam maior eficiência produtiva em relação a qualquer outro ruminante doméstico. Entre as décadas de 80 e 90 houve aumento de $51,6 \%$ na produção nacional, indicando o crescente mercado e interesse na atividade. 
SANTOS, C.M.S. e SANTANA, A.F. Produção de leite e duração da lactação de cabras da raça Pardo-alpina no município de Amélia Rodrigues - BA. PUBVET, Londrina, V. 5, N. 25, Ed. 172, Art. 1162, 2011.

No último censo agropecuário (IBGE, 2009a) o total de caprinos no Brasil foi 7.107.608, com um aumento de 516.962 cabeças nos últimos 10 anos. Já produção de leite da espécie foi de 35740188 litros, e a Bahia com seus 2.139 .136 de cabeças produziu aproximadamente $33,32 \%$ desse total.

O município de Amélia Rodrigues se localiza na mesorregião metropolitana de Feira de Santana-BA, possui 24.491 habitantes e um efetivo de 444 cabeças de caprinos de acordo com a Produção Pecuária Municipal de 2008 (IBGE, 2009b).

\section{REVISÃO DE LITERATURA}

As raças européias, de maneira geral, são especializadas para produção de leite, externando todo seu potencial quando exploradas em regiões de clima temperado. No Brasil, cabras das raças Parda Alpina, Saanen e Toggenburg têm uma grande aceitação na caprinocultura (GONÇALVES et al. 2001).

Essas três raças foram comparadas em mesmo sistema de criação no Distrito Federal por Soares Filho et al. (2001), e segundo eles a raça Saanen teve produção média diária de $2,34 \mathrm{Kg}$ de leite e duração da lactação entre cerca de 238,43 dias. Para esses valores a raça Parda Alpina teve, respectivamente, $2,01 \mathrm{Kg}$ leite/dia e 238,88 dias, enquanto que a Toggenburg foi de $1,70 \mathrm{Kg}$ leite/dia e 211,88 dias.

No entanto a inserção de genótipos exóticos de aptidão leiteira em condições adversas no semi-árido, altas temperaturas e radiação solar provocam estresse térmico nos animais com conseqüente diminuição da produção de leite, pois as cabras diminuem a ingestão de alimentos e aumentam o consumo de água (BRASIL et al., 2000).

Trabalhando com Pardo Alpina em sistema de confinamento, Sarmento et al. (2006) observaram que primíparas produziram em média 2,22 $\mathrm{Kg}$ de leite durante a primeira lactação e $2,12 \mathrm{Kg}$ de leite dentre todas as lactações 
SANTOS, C.M.S. e SANTANA, A.F. Produção de leite e duração da lactação de cabras da raça Pardo-alpina no município de Amélia Rodrigues - BA. PUBVET, Londrina, V. 5, N. 25, Ed. 172, Art. 1162, 2011.

observadas. No entanto, mestiças dessa raça com Moxotó em clima semi-árido apresentaram produção média de $800 \mathrm{~g}$ de leite durante a 1 a lactação segundo Silva et al. (2002), ainda assim esse valor pode ser considerado execelente para a região.

Contudo, a idade da cabra ao parto é um importante fator que influencia na produção de leite. O que se explica é o fato de que as cabras nos dois primeiros ciclos (gestação- lactação) reprodutivos ainda estão em crescimento e parte dos nutrientes provenientes da alimentação é destinada ao desenvolvimento dos diversos tecidos corporais, inclusive o tecido secretor da glândula mamaria em detrimento da produção de leite (GONÇALVES et al., 2001).

A relação entre produção de leite por dia e duração da lactação expressa-se crescente até certo limite, pois, a medida que avança o período lactífero as cabras produzem menos leite que inicialmente, expressando-se em uma reação quadrática, ou seja, a produção diária de leite não aumenta linearmente com o avanço do período de lactação. No entanto, a correlação genética entre essas duas características é alta indicando que grande parte dos efeitos genéticos aditivos age sinergicamente em ambas as características, resultado que sugere que a seleção de cabras com maior produção de leite levaria a lactações mais longas (PIMENTA FILHO et al., 2004).

Objetivou-se com este trabalho avaliar a produção e duração da lactação de cabras Parda Alpinas criadas a pasto com suplementação protéica no município de Amélia Rodrigues - BA.

\section{MATERIAL E MÉTODOS}

Os animais utilizados foram 20 cabras leiteiras da raça Pardo-Alpino e pertenceram a um criatório localizado na cidade de Amélia Rodrigues - BA. Os 
SANTOS, C.M.S. e SANTANA, A.F. Produção de leite e duração da lactação de cabras da raça Pardo-alpina no município de Amélia Rodrigues - BA. PUBVET, Londrina, V. 5, N. 25, Ed. 172, Art. 1162, 2011.

controles foram realizados diariamente, provenientes de ordenha mecânica, realizada duas vezes ao dia.

Os animais foram submetidos em regime a pasto e receberam concentrado e mineralização como suplementação. Foram devidamente vermifugados 3 vezes por ano, sendo mantidos em aprisco suspenso.

Os dados foram coletados de 2003 a 2004 durante a primeira e segunda lactação. E para a análise estatística foi utilizado o software SPSS Statistics.

\section{RESULTADOS E DISCUSSÕES}

A média e o desvio padrão da quantidade média de leite produzido/dia, produção total de leite e duração da primeira lactação estão descritos na tabela 1. Ao passo que os dados referentes a segunda lactação são descritos na tabela 2.

Tabela 1: Média ( $\mu$ ) e desvio padrão (s) da Produção de leite e Duração da Lactação de 20 cabras na primeira lactação

\begin{tabular}{cccc}
\hline & $\begin{array}{c}\text { Média de Leite } \\
\text { produzido / } \\
\text { dia }\end{array}$ & $\begin{array}{c}\text { Produção } \\
\text { total (Kg) }\end{array}$ & $\begin{array}{c}\text { Duração da } \\
\text { lactação } \\
\text { (dias) }\end{array}$ \\
\hline $\boldsymbol{H}$ & 2,14 & 408,81 & 191,665 \\
$\mathbf{S}$ & 0,156945 & 35,6966 & 20,90765 \\
\hline
\end{tabular}

A média da produção total de leite foi $408,81 \pm 35,697 \mathrm{Kg}$ e $482,93 \pm$ $65,917 \mathrm{Kg}$ para a primeira e segunda lactação respectivamente. Esses valores foram semelhantes no estudo feito por Brito et al. (200) cabras Pardo Alpinas, obtendo-se média de $421,96 \mathrm{Kg}$ de leite produzidos por lactação. Por sua vez, a média da duração da lactação para a primeira lactação foi de 191,665 \pm 
SANTOS, C.M.S. e SANTANA, A.F. Produção de leite e duração da lactação de cabras da raça Pardo-alpina no município de Amélia Rodrigues - BA. PUBVET, Londrina, V. 5, N. 25, Ed. 172, Art. 1162, 2011.

20,907 dias, enquanto que na segunda lactação a média foi de $630 \pm 28,047$ dias.

Tabela 2: Média ( $\mu$ ) e desvio padrão (s) da Produção de leite e Duração da Lactação de 20 cabras na segunda lactação

\begin{tabular}{cccc}
\hline & $\begin{array}{c}\text { Média de Leite } \\
\text { produzido / } \\
\text { dia }\end{array}$ & $\begin{array}{c}\text { Produção } \\
\text { total (Kg) }\end{array}$ & $\begin{array}{c}\text { Duração da } \\
\text { lactação } \\
\text { (dias) }\end{array}$ \\
\hline $\boldsymbol{H}$ & 2,275 & 482,925 & 219,63 \\
$\mathbf{S}$ & 0,28631 & 65,91709 & 28,04741 \\
\hline
\end{tabular}

Os resultados obtidos tanto para a primeira quanto para a segunda lactação foram inferiores aos achados de Soares Filhos et. al (2001) que encontrou uma média de produção de 513,88 \pm 193,53Kg de leite e $238,88 \pm$ 71,68 dias na raça Pardo Alpina; Tholon (2000) que encontrou média de produção de 766,39Kg em cerca de 281 dias, e Ribeiro (1997) com 572,57 \pm $133,97 \mathrm{Kg}$ em 219,03 \pm 48,09 dias, ambos trabalhos com cabras Saanen. Porém foram superiores aos resultados de Rodrigues et al. (2006) que encontrou 315,93 Kg na primeira lactação e 351,28 Kg em cabras multíparas da raça Saanen.

Também foram superiores a trabalhos feitos no semi-árido brasileiro com mestiças Pardo Alpina, que apontam animais 1/2 Pardo Alpina X Gurguéia com média de produção total e duração de lactação de $257,7 \pm 54,7 \mathrm{~kg}$ e 185,5 \pm 46,9 dias, respectivamente (PIMENTA FILHO et al., 2004), e mestiças $1 / 2$ Pardo Alpina X Moxotó produzindo cerca de $167,30 \pm 16,68 \mathrm{Kg}$ de leite por lactação (SILVA et al., 2002).

Foi observado entre as duas lactações, um incremento significativo $(p<0,05)$ de $74,115 \mathrm{~kg}$ na produção total de leite e um aumento significativo $(p<0,05)$ de 27,965 dias na duração da lactação. Esses valores refletem a 
SANTOS, C.M.S. e SANTANA, A.F. Produção de leite e duração da lactação de cabras da raça Pardo-alpina no município de Amélia Rodrigues - BA. PUBVET, Londrina, V. 5, N. 25, Ed. 172, Art. 1162, 2011.

influência da ordem de parto sobre a produção de leite e duração da lactação, cabras primíparas não estão fisiologicamente maturas quanto as pluríparas além de serem mais exigentes nutricionalmente (SILVA et al., 2002).

No entanto, o aumento da média de produção diária de 2,14 $\pm 0,157$ $\mathrm{Kg}$ (Tabela 1) para 2,275 $\pm 0,286 \mathrm{Kg}$ de leite (Tabela 2) não foi significativo $(p>0,05)$. Silva et al. (2009), apesar de terem encontrado médias de produção diária inferiores $(1,37 \mathrm{Kg}$ leite/dia na primeira e $1,39 \mathrm{Kg}$ leite/dia na segunda lactação) também não encontraram diferença estatística. Já os estudos com cabras da raça Saanen elaborados por Rodrigues et al. (2006) apesar de encontrarem médias semelhantes aos nossos achados $(2,19 \mathrm{Kg} /$ dia na primeira lactação e $2,34 \mathrm{Kg} /$ dia na segunda lactação), observaram aumento significativo $(p<0,05)$ na quantidade de leite tanto diária quanto total entre primíparas e multíparas.

Nesse trabalho também foram estudadas as correlações entre as variáveis duração e produção na primeira e segunda lactação e foi observado uma alta correlação positiva nas mesmas, sendo sua representatividade de 0,753 e 0,546, respectivamente. Esses dados foram semelhantes aos obtidos por Brito et al. (2009) com 0,65 com cabras Alpinas. Porém foram inferiores aos encontrados por Tholon (2000), estudando cabras Saanen que obteve correlação de 1,00; e Pimenta Filho et al. (2004) com correlação de 0,96 em cabras mestiças Pardo AlpinaXGurguéia. Por outro lado foram superiores aos de Ribeiro (1997), correlação de 0,43 com cabras Saanen.

\section{CONCLUSÕES}

Não foi observado aumento significativo na média de produção diária das cabras estudadas, e com base nisso o aumento significativo entre a primeira e segunda lactação na produção total de leite se deve principalmente ao aumento significativo na duração da lactação. 
SANTOS, C.M.S. e SANTANA, A.F. Produção de leite e duração da lactação de cabras da raça Pardo-alpina no município de Amélia Rodrigues - BA. PUBVET, Londrina, V. 5, N. 25, Ed. 172, Art. 1162, 2011.

Esse aumento é favorável e preferível em relação ao aumento da produção de leite diária, pois cabras que produzem mais leite por dia têm maiores tendências a terem mastite e têm maiores necessidades nutricionais.

Houve alta correlação positiva entre as variáveis produção leiteira e duração da lactação. Isso indica que a seleção para a duração da lactação poderia ser feita indiretamente pela seleção para produção total de leite e viceversa.

\section{REFERÊNCIAS}

BRASIL, L. H. A.; WECHESLER, F. S.; JÚNIOR, F. B. GONÇALVES, H. C. BONASSI, I. A. Efeitos do estresse térmico sobre a produção, composição química do leite e respostas

termorreguladoras de cabras da raça Alpina. Revista Brasileira de Zootecnia, Viçosa, v. 29, n. 6, p. 1632-1641, dez. 2000.

BRITO, L.F.; SILVA, F.G.; COSTA, E.V.; MELO, A.L.P.; MENEZES, G.R.O.; PEREIRA, R.J.; TORRES, R.A.; RODRIGUES, M.T. Parâmetros genéticos de características produtivas em caprinos da raça alpina. In: CONGRESSO INTERNACIONAL DE ZOOTECNIA, 11., 2009, Águas de Lindóia. Anais... Águas de Lindóia: INSA , 2009. p.1-4.

GONÇALVES, H. C.; SILVA, A. M.; WECHESLER, F. S.; RAMOS, A. A. Fatores genéticos e de meio na produção de leite de caprinos leiteiros. Revista Brasileira de Zootecnia, Viçosa, v. 30, n. 3, p.719-729, maio/jun. 2001.

GONÇALVES, H.C.; SILVA, A.M.; WECHESLER, F.S.; RAMOS, A.A.; PULZ, L.M.; LOSI, T.C. Parâmetros e Tendência Genética da Produção de Leite de Cabra no Brasil. Revista Brasileira de Zootecnia, Viçosa, v.31, n.6, p.2204-2208, nov./dez. 2002.

IBGE. Censo agropecuário 2006. Rio de Janeiro, Ed IBGE: 2009a. 777 p.

IBGE. Produção da Pecuária Municipal 2008. Rio de Janeiro, 2009b. Disponível em: <http://www.ibge.gov.br/cidadesat/topwindow.htm?1> Acesso em: 03.jun.2010

PIMENTA FILHO, E. C.; SARMENTO J. L. R.; RIBEIRO M. N. Efeitos genéticos e ambientais que afetam a produção de leite e duração da lactação de cabras mestiças no estado da Paraíba.

Revista Brasileira de Zootecnia, Viçosa, v.33, n.6, p.1426-1431, nov./dez. 2004.

QUADROS, D. G. Leite de cabra: produção e qualidade. PUBVET, Londrina, v. 2, n. 1, Ed. 12, Art. 363, 2008.

RIBEIRO, A.C. Estudo dos efeitos genéticos e de ambiente sobre características de importância econômica em caprinos da raça Saanen. 1997. 133p. Dissertação (Mestrado em Zootecnia) - Faculdade de Ciências Agrárias e Veterinárias, Universidade Estadual Paulista, Jaboticabal, 1997.

RODRIGUES, L.; SPINA, J.R.; TEIXEIRA, I.A.M.A.; DIAS, A.C.; SANCHES, A.; RESENDE, K.T. Produção, composição do leite e exigências nutricionais de cabras Saanen em diferentes 
ordens de lactação. Acta Scientiarum Animal Sciences, Maringá, v. 28, n. 4, p. 447-452, out./dez. 2006.

SARMENTO, J. L. R.; REIS FILHO, J. C.; ALBUQUERQUE, L. G.; LOPES, P. S.; RODRIGUeS M. T. Avaliação genética de caprinos da raça Alpina utilizando-se a produção de leite no dia do controle. Revista Brasileira de Zootecnia, Viçosa, v.35, n.2, p.443-451, mar./abr. 2006.

SILVA, V. N.; RANGEL, A. H. N.; BRAGA, A. P.; MAIA, M. S.; MEDEIROS, H. R. Influência da raça, ordem e ano de parto sobre a produção de leite caprino. Acta Veterinaria Brasilica, v.3, n.4, p.146-150, 2009.

SILVA, F. L. R.; ANDRADE, V. O.; LIMA, F. A. M. Produção de leite de cabra $1 / 2$ Pardo Alpina X Moxotó no Estado do Ceará. Revista Científica de Produção Animal, v. 4, n. 1-2, p. 77-82, 2002.

SOARES FILHO, G.; McMANAUS, C.; MARIANTE, A. S. Fatores genéticos e ambientais que influenciam algumas características de reprodução e produção de leite em cabras no Distrito Federal. Revista Brasileira Zootecnia, Viçosa, v. 30, n. 1, p.133-140, jan./fev. 2001.

THOLON, P. Estudo genético quantitativo de características de importância econômica em caprinos da raça Saanen. 2000. 55p. Dissertação (Mestrado em Zootecnia) - Faculdades de Ciências Agrárias e Veterinárias, Universidade Estadual Paulista, Jaboticabal, 2000. 